\title{
Synthese neuer organischer Schwefelverbindungen aus aromatischen Thiolverbindungen und Sulphinsäuren
}

\author{
Jewgeni M. Alow, Nina P. Gerassimowa, Pjotr W. Korikow, Juri A. Moskwitschjow, \\ Nikolai A. Noshnin, Alexej N. Paschinin
}

Die rasche Entwicklung der Chemie organischer Schwefelverbindungen in der zweiten Hälfte des 20. Jahrhunderts wurde durch drei wichtige Prozesse ausgelöst:

- die Einbeziehung von schwefelhaltigen und stark schwefelhaltigen Erdölen sowie die Verwendung von schwefelhaltigen Abfällen zur Steigerung der Produktion, die durch die Entwicklung anderer Industriebranchen notwendig wurde;

- die Erweiterung der Anwendungsbereiche von organischen Schwefelverbindungen bei der Produktion von Arzneimitteln, Pestiziden, Flotationsmitteln, Zusatzstoffen für Öle und Heizstoffe, Inhibitoren gegen Metallkorrosion, Polymerisationsreglern, Farbstoffen u. a.;

- durch das große Interesse an organischen Schwefelverbindungen als Modelle für die Erforschung vieler wichtiger Grundlagenkonzeptionen in der organischen Chemie.

Aus diesem Grunde ist die Synthese neuer organischer Schwefelverbindungen mit potenziell nützlichen Eigenschaften sowie die Untersuchung der Gesetzmäßigkeiten des Reaktionsverlaufs mit dem Ziel, diese Prozesse bewusst zu steuern, von besonderer Aktualität. In der vorliegenden Arbeit wurden Reaktionen der nukleophilen Addition von aromatischen Thiolverbindungen und Sulfinsäuren an aktivierte Doppelbindungen (Vinylketone und Akrylnitrile) und die weitere Umwandlung der entstandenen Addukte zu heterozyklischen Verbindungen untersucht. Das Anwendungsgebiet der letztgenannten Stoffe ist bekanntermaßen sehr groß [1]: Ihr Anteil unter Arzneimitteln und Präparaten, die in der Landwirtschaft und Veterinärmedizin eingesetzt werden, ist dominierend. Sie werden als optische Aufheller, Antioxidanten, Inhibitoren gegen Metallkorrosion und diverse Zusatzstoffe angewandt. Viele Farbstoffe und Pigmente besitzen eine heterozyklische Struktur.

\section{Die Synthese von schwefelorganischen Deri- vaten der ungesättigten $\alpha$ - und $\beta$-Ketone}

Ungesättigte $\alpha$ - und $\beta$-Karbonylverbindungen sind aufgrund ihres hohen Reaktionsvermögens gut geeignet zur Herstellung diverser Derivate und werden in der organischen Synthese häufig verwendet. Das ist bedingt durch die Existenz gekoppelter Mehrfachbindungen $(\mathrm{C}=\mathrm{C}-\mathrm{C}=\mathrm{O}) \mathrm{mit}$ delokalisierter Elektronendichte und ambidenter Elektrophilie in den Molekülen dieser Sintone. In der letzten Zeit ist das Interesse an der Chemie der ungesättigten $\alpha$ - und $\beta$-Ketone, insbesondere der 1,3-diarylsubstituierten Propenone (Chalkone) aufgrund der Möglichkeit, auf deren Basis biologisch aktive Stoffe mit einem breiten Wirkungsspektrum zu erzeugen $[2,3]$, deutlich gewachsen. Dazu zählen Arzneimittel, Bakterizide, Insektizide und Polymere [4] speziell für den Einsatz in der Optik [5] und andere fuir die Praxis wertvolle Produkte. Eine Reihe von ungesättigten aromatischen Oxiketonen (Butein, Kartamin, Kurkumin), die Naturpigmente darstellen und als Farbstoffe für Baumwolle und Seide eingesetzt werden, werden als Indikatoren genutzt [6].

Dennoch fehlen in der Literatur bis heute systematische Untersuchungen zur Kinetik und zum Mechanismus einer z. B. für die Praxis so wichtigen Reaktion wie Thiolanlagerung an $\alpha, \beta$-ungesättigte Ketone, die zur Bildung von Sulfiden führt, den potenziellen Reglern der Polymerisationsprozesse, Vulkanisierungsbeschleuniger, Insektizide, Arzneimittel u. a. [7]. Die Synthese von $\boldsymbol{\gamma}$-Ketosulphonen auf der Grundlage der Chalkone wurde nur in wenigen Publikationen behandelt $[8,9]$, obwohl die Reaktionsprodukte potenzielle Schutzstoffe gegen radioaktive Strahlung und Arzneipräparate zur Behandlung von Malaria und Tuberkulose darstellen.

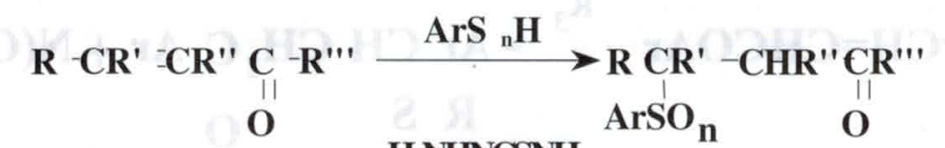

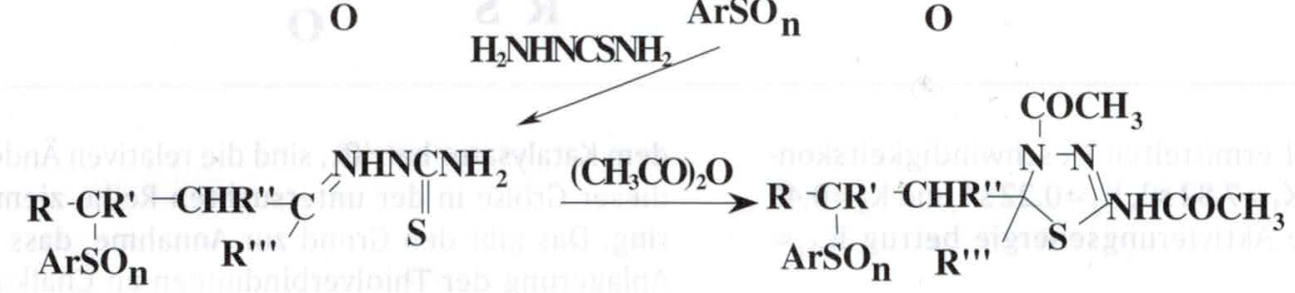

$$
\begin{aligned}
& \mathrm{n}=0,2 ; \mathrm{Ar}=4-\mathrm{CH}_{3} \mathrm{C}_{6} \mathrm{H}_{4}, 4-\mathrm{BrC}_{6} \mathrm{H}_{4}, 3,4-\mathrm{Cl}_{2} \mathrm{C}_{6} \mathrm{H}_{3}, 2,5-\mathrm{Cl}_{2} \mathrm{C}_{6} \mathrm{H}_{3}, 4-\mathrm{CH}_{3} \mathrm{CONHC}_{6} \mathrm{H}_{4} \text {, } \\
& 3-\mathrm{NO}_{2} \mathrm{C}_{6} \mathrm{H}_{4} ; \mathrm{R}=\mathrm{H}, \mathrm{CH}_{3}, \mathrm{C}_{6} \mathrm{H}_{5}, 2-\mathrm{HOC}_{6} \mathrm{H}_{4}, 2-\mathrm{C}_{4} \mathrm{H}_{3} \mathrm{O} ; \mathrm{R}^{\prime}=\mathrm{H}, \mathrm{CH}_{3} ; \mathrm{R}^{\prime \prime}=\mathrm{H} \text {; } \\
& \mathbf{R}^{\prime \prime \prime}=\mathrm{CH}_{3}, \mathrm{C}_{6} \mathrm{H}_{5} ; \mathbf{R}^{\prime \prime}+\mathrm{R}^{\prime \prime}=\left(\mathrm{CH}_{2}\right)_{4} \text {. }
\end{aligned}
$$


Die ungesättigten $\alpha, \beta$-Ketone als Ausgangsstoffe haben wir nach der Claisen-Kondensation synthetisiert. In der vorliegenden Arbeit wurden die Reaktionen der nukleophilen 1,4-Addition von aromatischen Thiolverbindungen und Sulphinsäuren an diese Substrate sowie die nachfolgende Kondensation der gesättigten Ketone unter Einbeziehung der Karbonylgruppe mit Hilfe von Thiosemikarbazid und Essigsäureanhydrid untersucht.

Die kinetischen Untersuchungen der Thiolanlagerung der Enonverbindungen haben wir am Beispiel der Anlagerung des 4-Methylthiophenols an 1,3-Diphenyl-1Propen-3-on (Chalkon) in reinem Methanol mit Triethylamin als Katalysator durchgefuihrt. Die kinetischen Messungen erfolgten spektrophotometrisch nach der optischen Dichte des Reaktionsgemischs bei einer Wellenlänge von $\lambda_{\max }=380 \mathrm{~nm}$, die der Absorption von Chalkon entspricht (Abb. 1).

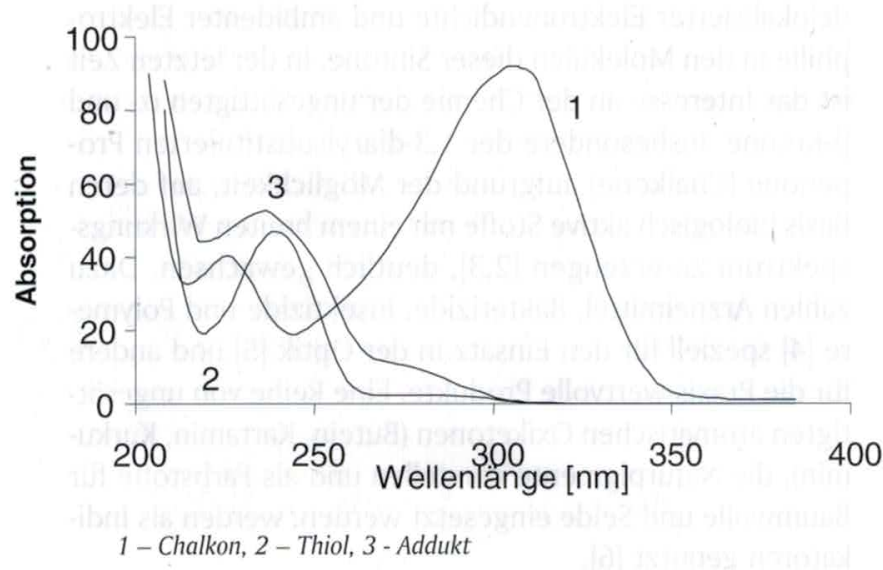

Abb. 1: Absorptionsspektren der Reaktionsstoffe und des Addukts.

Es wurde nachgewiesen, dass die zu untersuchende Reaktion eine biomolekulare katalytische Reaktion ist, deren Mechanismus mit Hilfe des folgenden Schemas dargestellt werden kann: führt. In Bezug auf ihre elektronischen Eigenschaften variierten die Substituenten von stark ausgeprägten Donatoren bis zu starken Akzeptoren (Tabelle 1).

\begin{tabular}{l|l|l|l|l|l}
$R$ & \multicolumn{2}{|l|}{\begin{tabular}{l} 
Energie der höchstbesetzten \\
\multicolumn{2}{l}{ Molekülorbitale, V }
\end{tabular}} & \multicolumn{2}{|l|}{ Atomladung S, y. e. } & $\begin{array}{l}\text { W·10.5, } \\
\mathrm{mol} / \mathrm{dm}^{3} / \mathrm{s}\end{array}$ \\
\hline & $\begin{array}{l}\text { Thiol- } \\
\text { verbindung }\end{array}$ & $\begin{array}{l}\text { Komplex- } \\
\text { verbindung } \\
\text { mit Thiol }\end{array}$ & Thiol & $\begin{array}{l}\text { Komplex- } \\
\text { verbindung } \\
\text { mit Thiol }\end{array}$ & \\
\hline $\mathrm{CH} 30$ & -8.52 & -8.09 & 0.095 & -0.093 & 1.28 \\
\hline $\mathrm{CH} 3$ & -8.67 & -8.28 & 0.097 & -0.094 & 0.92 \\
\hline $\mathrm{H}$ & -8.78 & -8.31 & 0.098 & -0.097 & 0.50 \\
\hline $\mathrm{Cl}$ & -8.78 & -8.34 & 0.105 & -0.087 & 0.35 \\
\hline $\mathrm{Br}$ & -8.94 & -8.48 & 0.107 & -0.087 & 0.13 \\
\hline $\mathrm{N} 02$ & -9.52 & -9.03 & 0.0135 & -0.068 & - \\
\hline
\end{tabular}

Tabelle 1: Ergebnisse der quantenchemischen Berechnungen für $4 R$-substituierte aromatische Thiolverbindungen und die Anfangsgeschwindigkeiten ihrer Reaktion mit Chalkonen.

Vertreter der untersuchten Reihe von Thiolverbindungen reagieren nicht mit Chalkonen ohne Katalysator. Die von uns nach der Methode RM-3 durchgefuihrten quantenchemischen Berechnungen der Reaktantenmodelle zeigen, dass die Werte der Energie der höchstbesetzten Molekiilorbitale von Thiolverbindungen (Tab. 1) und Chalkonen $(E=-9,37 \mathrm{eV})$ ziemlich nah beieinander liegen. Das ist wahrscheinlich der Hauptfaktor, der die Reaktion des Chalkons mit nichtaktivierten Thiolverbindungen verhindert. Die Komplexbildung mit Triethylamin erhöht die Anregungsenergie der höchstbesetzten Molekuilorbitale und ermöglicht den Reaktionsablauf mit dem Chalkon.

In Tabelle 1 sind die Thiolverbindungen nach der Abnahme der Anfangsgeschwindigkeit ihrer Reaktion mit Chalkonen geordnet. Dieser Abnahme entspricht die Verringerung der höchstbesetzten Molekülorbital der Thiolverbindungen und deren Komplexe mit dem Triethylamin. Was die Ladung der Schwefelatome in den Thiolverbindungen und in ihren Komplexen mit

$$
\begin{aligned}
& \mathrm{RSH}+\mathrm{N}\left(\mathrm{C}_{2} \mathrm{H}_{5}\right)_{3} \underset{\mathrm{k}_{2}}{\stackrel{\mathrm{k}_{1}}{\rightleftarrows}} \mathrm{RSH} \cdot \mathrm{N}\left(\mathrm{C}_{2} \mathrm{H}_{5}\right)_{3} \\
& \mathrm{RSH} \cdot \mathrm{N}\left(\mathrm{C}_{2} \mathrm{H}_{5}\right)_{3}+\mathrm{ArCH}=\mathrm{CHCOAr} \\
& \mathbf{k}_{3} \\
& >\mathrm{Ar} \mathrm{CH} \mathrm{CH} \mathrm{CH}_{2} \mathrm{Ar}+\mathrm{N}\left(\mathrm{C}_{2} \mathrm{H}_{5}\right)_{3} \\
& \text { R S } \\
& \text { O }
\end{aligned}
$$

Die experimentell ermittelten Geschwindigkeitskonstanten betrugen $\mathrm{K}_{1}=7,82 \mathrm{~s}^{-1}, \mathrm{~K}_{2}=0,22 \mathrm{~s}^{-1}$ und $\mathrm{K}_{3}=0,40$ $\mathrm{dm}^{3} \mathrm{~mol}^{-1} \mathrm{~s}^{-1}$, die Aktivierungsenergie betrug $\mathrm{E}_{\mathrm{akt}}=$ $42,414 \mathrm{~kJ} / \mathrm{mol}$.

Um den Einfluss der Substituente in aromatischen Thiolverbindungen auf die Geschwindigkeit ihrer Reaktion mit dem Chalkon zu untersuchen, wurde eine Reihe von Versuchen mit verschiedenen Thiolverbindungen durchge- dem Katalysator betrifft, sind die relativen Änderungen dieser Größe in der untersuchten Reihe ziemlich gering. Das gibt den Grund zur Annahme, dass die 1,4Anlagerung der Thiolverbindungen an Chalkone eine durch die Atombahnen kontrollierte Reaktion darstellt. Zur gleichen Schlussfolgerung sind Autoren, die die Thiolanlagerung an Azetylketone [10] und Anlagerung von $\mathrm{CH}$-Säuren an Vinylketone untersucht haben, gekommen [11]. 
Wir haben die Synthesebedingungen für $\beta$-Arylthioketon so gewählt, dass der Ertragswert möglichst nah an der quantitativen Ausbeute liegt. Die Ausbeute von $\beta$-Arylsulphonylketonen war deutlich geringer als die der $\beta$ Arylthioketone und betrug $36-80 \%$. Das ist offensichtlich auf die niedrigere Nucleophilie des Sulfinat-Anions im Vergleich zum Thiolat, sowie auf mögliche räumliche Probleme bei der Anlagerung des voluminösen SulfinatAnions zurückzuführen. Im Gegensatz zu den Thiolverbindungenn lagern sich die Arensulfinsäuren an ungesättigte $\alpha, \beta$-Ketone erst nach der Aktivierung der Karbonylgruppe an. Die Aktivierung erfolgt in der Regel durch eine Protonierung $\left(\mathrm{CH}_{3} \mathrm{COOH}, \mathrm{H}_{3} \mathrm{BO}_{3}, \mathrm{HCL}\right.$ u. a.).

Der Aufbau der synthetisierten Verbindungen wurde durch die Infrarotspektroskopie und paramagnetische Resonanz nachgewiesen. Die Infrarotspektren der synthetisierten Verbindungen weisen Absorptionsmaxima im Bereich $1630-1660 \mathrm{~cm}^{-1}(\mathrm{C}=0)$ auf. Bei der paramagnetischen Resonanz gibt das Proton der Methingruppe ein Signal in Form eines Multipletts im Bereich 3,0-3,5 ppm. Die Protonsignale der Ethylgruppe, die in Bezug auf die Ketongruppe eine $\alpha$-Position hat, liegen im Bereich 2,3 2,5 ppm in Form eines Singuletts. Die Protonen des Furanrings strahlen Signale in Form eines Singuletts in den Bereichen 6,1, 6,3 und 7,5 ppm aus.

Die Untersuchung der Kondensation der gewonnenen ßAryl-thio(sulphonyl)ketone mit Thiosemicarbazid in Alkohol hat ergeben, dass $\alpha$-arylsubstituierte Ketone wesentlich langsamer als $\alpha$-Methylketone reagieren. Das ist offensichtlich auf die sterischen Faktoren zurückzuführen. Ferner entstehen neben Thiosemikarbazonen auch eine Reihe von Nebenprodukten, die eine Abscheidung der Zielprodukte wesentlich erschweren. Die Umwandlung der Thiosemikarbazonen in Thiadiazoline in einer Zyklisierungsreaktion in Essigurethanhydrid bei $100{ }^{\circ} \mathrm{C}$ und bei einer Dauer von zwei Stunden verläuft ziemlich gleichmäßig und mit hohem Ertrag.

Die Infrarotspektren der synthetisierten Verbindungen weisen Absorptionsmaxima in den Bereichen 1550 - 1580 $(\mathrm{C}=\mathrm{N}), 1630-1660(\mathrm{C}=0), 3200-3240(\mathrm{NH})$ auf. In den paramagnetischen Resonanzspektren der Thiosemikarbazonen, der Addukte von Benzolazeton und 4-Methylthiophenole strahlt das Proton der Methingruppe ein Signal in Form eines Multipletts im Bereich 4,7 - 5,5 ppm aus. In den paramagnetischen Resonanzspektren der 5-substituierten-2-Azetamino-4-azyl1-1,3,4-D22-Thiodiasoline strahlen die Protonen der Gruppe $\mathrm{CH}_{3} \mathrm{CO}$ Signale im Bereich 2,0-2,3 ppm aus, das $\mathrm{CH}_{3}$-Signal erscheint bei 2,4 ppm und das $\mathrm{CH}_{3}$-Signal erscheint bei $11,2-11,4 \mathrm{ppm}$.

\section{Synthese von heterozyklischen Derivaten der Arenthio(sulphonyl)karbonsäuren}

Unter organischen Schwefelverbindungen sind Arenthio(sulphonyl)karbonsäuren und deren Derivate (Nitril, Äther, Amid, Salze u. a.) von besonderer Bedeutung. Für die Synthese dieser Verbindungen wurden gute Methoden entwickelt [12]. Sie werden als Zwischenprodukte bei der Synthese von Farbstoffen, Herbiziden, Wachstumsreglern bei Pflanzen, Arzneipräparaten usw. verwendet [13]. Wir haben eine Reihe neuer Derivate der Arenthio(sulphonyl)karbonsäuren synthetisiert [14], die biologisch aktiv waren.

Unter den Derivaten der Karbonsäuren sind Nitrile von besonderem Interesse. Durch die stark polare Nitrilgruppe und ihre hohe Polarisierbarkeit unter Einwirkung verschiedener Stoffe können Nitrile in verschiedene Reaktionen treten, die u. a. auch zur Bildung von heterozyklischen Verbindungen fuihren.

Die Ausgangsnitrile haben wir durch Cyanethylierung von aromatischen Thiolverbindungen und Sulphinsäuren mit einer Ausbeute von über $90 \%$ synthetisiert:

$$
\operatorname{ArSO}_{n} \mathrm{H}+\mathrm{CH}_{2}=\mathrm{CH}-\mathrm{CN} \underset{\mathrm{n}=0,2}{\longrightarrow} \mathrm{ArSO}_{\mathrm{n}} \mathrm{CH}_{2} \mathrm{CH}_{2} \mathrm{CN}
$$

In der vorliegenden Arbeit wurden die Arenthio(sulphonyl)propionnitrile (1) zum ersten Mal für die Synthese von Benzimidazol (II, $x=N H$ ), Oxazolen (II, $x=0$ ), Thiazolen (II, $x=S$ ), Oxadiazolen /IV), Imidazolinen (V), s-Triazinen (VI) nach dem folgenden Schema verwendet:

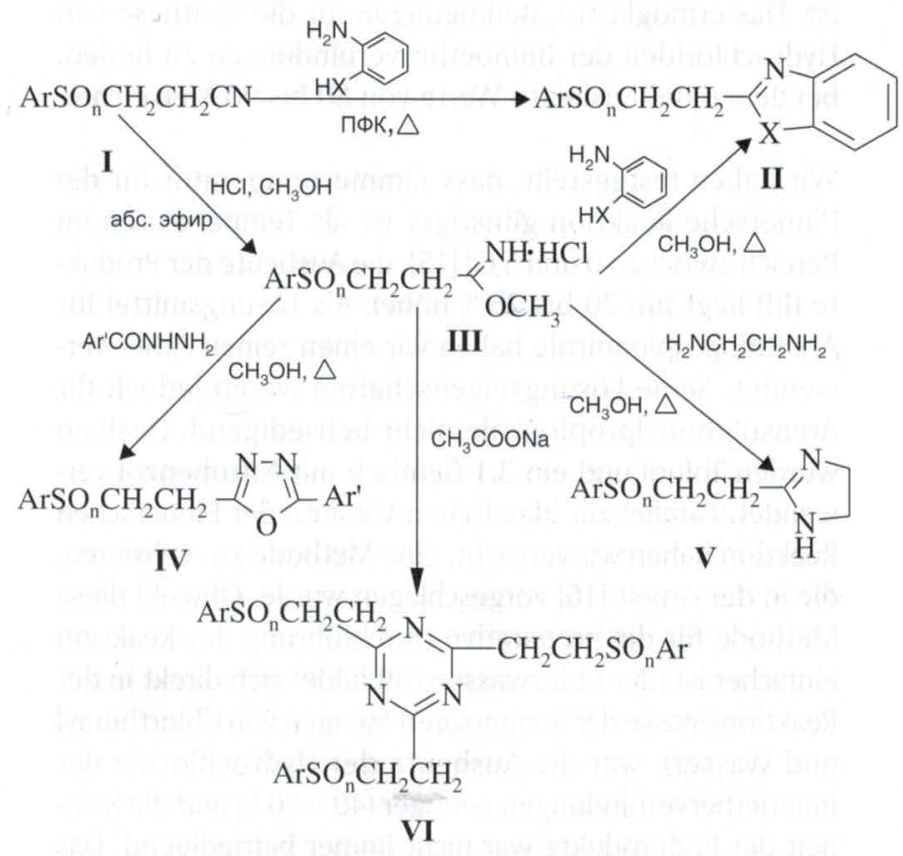

$\mathrm{n}=0,2 ; \mathrm{X}=\mathrm{NH}, \mathrm{S}, \mathrm{O} ; \mathrm{Ar}=4-\mathrm{CH}_{3} \mathrm{C}_{6} \mathrm{H}_{4}, 4-\mathrm{ClC}_{6} \mathrm{H}_{4}, 3,4-$ $\mathrm{Cl}_{2} \mathrm{C}_{6} \mathrm{H}_{3}, 4-\mathrm{BrC}_{6} \mathrm{H}_{4}, 4-\mathrm{CH}_{3} \mathrm{OC}_{6} \mathrm{H}_{4}, 2-\mathrm{C}_{10} \mathrm{H}_{7} ; \mathrm{Ar}=\mathrm{C}_{6} \mathrm{H}_{5}$, 4$\mathrm{BrC}_{6} \mathrm{H}_{4}, 4-\mathrm{FC}_{6} \mathrm{H}_{4}, 4-\mathrm{NC}_{5} \mathrm{H}_{4}$.

Es wurde die einstufige Gewinnung von Benzazolen (II) aus Arenthio(sulphonyl)propionnitril in der Polyphosphorsäure (PPS) als Medium bei einer Temperatur von $110-170{ }^{\circ} \mathrm{C}$ und einer Reaktionszeit von 2 bis 8 Stunden untersucht. Es wurde festgestellt, dass bei dieser Synthesemethode eine befriedigende Ausbeute (bis zu $70 \%$ ) von Verbindungen mit $n=2$ und $x=N H$ erreicht wird. Es ist erforderlich, dass die Temperatur im Bereich zwischen 150 und $170{ }^{\circ} \mathrm{C}$ liegt und die Reaktionszeit 6 Stunden beträgt. Bei der Synthese von Verbindungen mit $\mathrm{n}=2$ und $\mathrm{x}=0$, S beträgt die Ausbeute nicht mehr als 26 $-32 \%$ und für $\mathrm{n}=0$ und $\mathrm{x}=\mathrm{NH}$ liegen die entsprechen- 
den Werte zwischen 10 und $15 \%$, dabei kommt es zu einem starken Verharzen des Reaktionsgemisches. Die einstufige Kondensation in der Polyphosphorsäure als Medium kann also nur für die Synthese von 2-(2-Benzimidazolil)ethylarylsulphonen empfohlen werden.

Aus diesem Grunde haben wir eine andere Synthesemethode mit einer niedrigeren Temperatur gewählt, die auf einer Umwandlung der Arenthio(sulphonyl)propionnitrile in reaktionsfähigere Syntone - Methyliminoetherverbindungen der Arenthio(sulphonyl)propionsäuren und<smiles>COC(=NC=O)c1ccc([PH2+]NC(=O)CCOS(=O)(=O)c2ccc(C)cc2)cc1</smiles>

derer Hydrochloride(III) - basiert. Es wurde festgestellt, dass bei der Basenkatalyse $\left(\mathrm{CH}_{3}\right.$ in Methanol, $10 \mathrm{Gew} \%$ zum Nitril, bei $20^{\circ} \mathrm{C}$ und $64^{\circ} \mathrm{C}$ ), die fuir hochelektrophile Nitrilverbindungen empfohlen wird, die untersuchten Verbindungen keine Iminoetherverbindungen bilden. Zugleich binden sie leicht Alkohole bei Pinnerschen Reaktionsbedingungen, bei denen die Elektrophilie der Nitrilgruppe aufgrund der Bildung des Karbiminkations $\mathrm{RC}^{+}=\mathrm{NH}$ unter Einwirkung von Chlorwasserstoff erhöht ist. Das ermöglichte, Bedingungen für die Synthese von Hydrochloriden der Iminoetherverbindungen zu finden, bei denen die Ausbeute Werte von 80 bis $90 \%$ erreichte.

Wir haben festgestellt, dass Zimmertemperatur für die Pinnersche Reaktion günstiger ist als Temperaturen im Bereich zwischen 0 und $5 \mathrm{C}^{\circ}$ [15], die Ausbeute der Produkte (III) liegt um 20 bis $25 \%$ höher. Als Lösungsmittel für Arenthiopropionnitrile haben wir einen reinen Äther verwendet. Seine Lösungseigenschaften waren jedoch für Arensulphonylpropiontrile nicht befriedigend. Deshalb wurden Toluol und ein 3:1-Gemisch mit Nitrobenzol verwendet. Parallel zur klassischen Variante der Pinnerschen Reaktion haben wir versucht, eine Methode zu realisieren, die in der Arbeit [16] vorgeschlagen wurde. Obwohl diese Methode fuir die preparative Durchfuihrung der Reaktion einfacher ist (der Chlorwasserstoff bildet sich direkt in der Reaktionsmasse der äquimolaren Mengen von Chlorthionyl und Wasser), war die Ausbeute der Hydrochloride der Iminoetherverbindungen geringer $(40-70 \%)$ und die Reinheit der Endprodukte war nicht immer befriedigend. Das wurde offensichtlich durch Nebeneffekte verursacht, die durch die Wasserzugabe hervorgerufen wurden.

Die von uns synthetisierten Hydrochloride der Iminoetherverbindungen (III) waren einwandfreie Sintone fuir die Synthese angestrebten heterozyklischen Verbindungen. Die besten Ausbeuten (60 bis $92 \%$ ) für die Verbindungen (II, IV, V) wurden erzielt durch ein 4- bis 6-stündiges Kochen der äquimolaren Reagenzmengen in Methanol. Die Verbindungen (IV) wurden mittels der Reaktion des wasserfreien Natriumazetats mit Verbindungen (III) synthetisiert. Die Ausbeute betrug 43-60\%. Die Rolle des Natriumazetats in diesem Prozess besteht im Abspalten des Chlorwasserstoffs vom Iminesterhydrochlorid und der Bildung von Essigsäure, die ein Katalysator für die Trimerisation der Iminoäther darstellt:

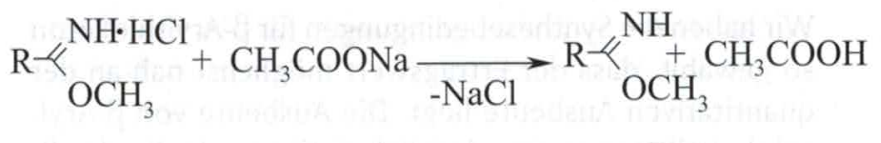<smiles>[R]OC([R])NCC</smiles>

Das Oxadiazol (IV, $\mathrm{Ar}=4-\mathrm{CH}_{3} \mathrm{C}_{6} \mathrm{H}_{4}, \mathrm{Ar}=\mathrm{C}_{6} \mathrm{H}_{5}, \mathrm{n}=2$ ) wurde außerdem mittels Kontrollsynthese synthetisiert:

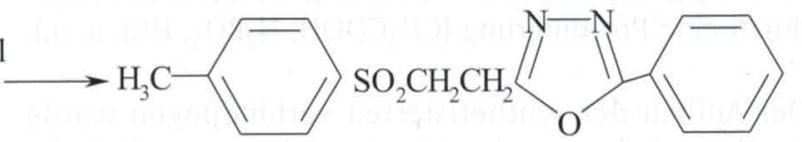

Die Verbindungen (II, IV), die mittels zweier Methoden synthetisiert wurden, hatten gleiche Schmelzpunkte und identische Spektralcharakteristika.

Die Infrarotspektren der Verbindungen (II, IV, V, VI) haben Absorptionsmaxima in den Bereichen 16530 $1540 \mathrm{~cm}^{-1}(\mathrm{C}=\mathrm{N})$. Die Absorptionsmaxima der Verbindungen (II) und (IV) liegen im Bereich 1035-1080 $\mathrm{cm}^{-1}$ (C$\mathrm{O}-\mathrm{C})$, die für Schwingungen der Gruppe $-\mathrm{SO}_{2}$ charakteristisch sind. In den paramagnetischen Resonanzspektren der Verbindungen (II, IV, V, VI) sind die Protonen der Methingruppen in Form von Tripletten als zwei Gruppen von Signalen im Bereich 3.0 - 3.2 und 3.5 3.7 ppm sichtbar.

\section{Literatur}

[1] Джилкрист Т. Химия гетероциклических соединений: пер. с англ.- М.: Мир, 1996.- 464 с., ил. (Gilchrist, T.: Chemie der heterozyklischen Verbindungen. Übersetzung aus dem Englischen, Moskau, Verlag „Mir“. S. 464)

[2] Dimmrok J. R., Patil S. A., Sardessai M. S., Mazurek M.: Pharmazin. 1987. Vol. 42, № 2.- P. 111-113.

[3] Пат. 57-197262 Япония. Способ получения оптически активных b-арилтио- и алкилтиокетонов. (Patent 57 197262, Japan. Verfahren zur Synthese optisch aktiver $\beta$ Arylthio- und Alkylthioketone.)

[4] Сидорович А.В., Баклагина Ю.Г., Лукасов С.В., Лаврентьев В.К. и др. Журн. Прикл. химии.- 1998. - т. 71, № 9.- C. 1500-1504. (Sidorowitsch, A. B., Baklagina, Ju. G., Lukassow, S. W., Lawrentjew, W. K. u. a.: Journal für angewandte Chemie, Band 71, Nr. 9, S. 1500-1504.)

[5] Пат. 5097016 США, МКИ С 07 К 7/10. Nonlinear optical materials of polypeptides. (Patent 5097016, USA.)

[6] Каррер П. Курс органической химии.-Л.: Государственное научно-техническое издательство химической литературы, 1960.-1216c. (Kapper, P.: Handbuch der organischen Chemie, Leningrad, Staatlicher Verlag für chemische Literatur, 1960, S. 1216)

[7] Прилежаева Е. Н., Шостаковский М.Ф. Успехи химии. - 1963.-№ 8.-C. 908-911. (Prileshajewa, E. N., Schostakowski, M. F.: Erfolge der Chemie, 1963, Nr. 8, S. 908-911.)

[8] Gilman H., Cason F. J. Am. Chem. Soc.-1950.- Vol. 72, № 8.-P 146-148. 
[9] Шабаров Ю. С. Органическая химия. Т. 1. Нециклические соединения: Учебник для вузов - 2-ое изд., перераб. - М.: Мир, 1996.-496 c. (Schabarow, Ju. S.: Organische Chemie, Band 1. Nicht-zyklische Verbindungen. Lehrbuch für Hochschulen, 2. überarbeitete Aufl., 1996, S. 496)

[10] Бодриков И. В., Коршунов С. П., Бажан Л. И. Журн. Орган. Химии.- 1988.- № 4.- С. 679-683. (Bodrikow, I. W., Korschunow, S. P. und Bashan, L. I.: Journal für Organische Chemie, 1988, Nr. 4, S. 679-683.)

[11] Яновская Л. А., Крышталь Г. В., Кульганек В. В. // Успехи химии.- 1984.-№ 8.- С. 1280. (Janowskaja, L. A. Kryschtal, G. W. und Kulganew, W. W.: Erfolge der Chemie, 1984, Nr. 8, S. 1280.)

[12] Мирскова А.Н., Крюкова Г.Г., Левковская Г. Г., Гусева С.А., Воронков М.Г. Журн. Орган. Химии.- 1984.- Е. 20, № 5.- C. 602-608. (Mirskowa, A. N., Krjukowa, G. G., Lewkowskaja, G. G., Gussewa, S. A. und Woronkow, M. G.: Journal für organische Chemie, 1984, E 20, Nr. 5, S. 602608.)

[13] Omokawa H., Ichizen N., Tabogami S., Takematsu №. Agric. Biol. Chem.- 1985.- Vol. 49, 12.- P. 3551-3555.

[14] Пат. СССР 1806134. Трис-(2-оксиэтил)аммониевая соль3,4-дихлорфенилсульфонилуксусной кислоты в качестве стимулятора корнеобразования/ Алов Е.М., Новиков С.Э., Никифоров А.В., Москвичев Ю.А. и др. (Patent der UdSSR 1806134: Tris-(2-Oxiethyl)ammoniumsalz der 3,4-Dichlorphenylessigsäure als Stimulator der Wurzelbildung, Alow, E. M., Nowikow, S. E., Nikiforow, A. W, Moskwitschjow, Ju. A. u. a.)

[15] Зильберман Е.И. Реакции нитрилов. М: Химия.-1972. -448 c. (Silberman, E. I.: Nitrilreaktionen, Moskau, Verlag Chemie, 1972, S. 448)

[16] А. с. СССР 115896 Зарецкий В.И.: Бюлл. изобр., 1958.№ 11.-C. 28 (Urheberschein der UdSSR 115896 von Sarezki, W. 1.. In: Bulletin der Erfindungen, 1958, Nr. 11, S. 28.)

\section{Autoren}

\section{Jewgeni M. Alow}

Doktor der Chemiewissenschaften

Leiter des Lehrstuhls für organische Chemie

\section{Nina P. Gerassimowa}

Kandidat der chemischen Wissenschaften wissenschaftliche Mitarbeiterin am Lehrstuhl für chemische Technologie organischer Stoffe

\section{Pjotr W. Korikow}

Aspirant am Lehrstuhl für chemische Technologie organischer Stoffe

\section{Juri A. Moskwitschjow}

Professor, Doktor der Chemiewissenschaften Rektor der Jaroslawler staatlichen technischen Universität

Leiter des Lehrstuhls für chemische Technologie organischer Stoffe, verdienter Wissenschaftler der Russischen Föderation

\section{Nikolai A. Noshnin}

wissenschaftlicher Mitarbeiter am Lehrstuhl für chemische Technologie organischer Stoffe

\section{Alexej N. Paschinin}

Aspirant am Lehrstuhl für chemische Technologie organischer Stoffe

\section{Übersetzung:}

\section{Dr. Olga Rösch}

Technische Fachhochschule Wildau

Sprachenzentrum

Tel. (0 33 75) 508-367

E-Mail: roesch@sprz.tfh-wildau.de 